\title{
Individual differences in fixation duration distributions in reading
}

\author{
Adrian Staub • Ashley Benatar
}

Published online: 1 May 2013

(C) Psychonomic Society, Inc. 2013

\begin{abstract}
The present study investigated the relationship between the location and skew of an individual reader's fixation duration distribution. The ex-Gaussian distribution was fit to eye fixation data from 153 subjects in five experiments, four previously presented and one new. The $\tau$ parameter was entirely uncorrelated with the $\mu$ and $\sigma$ parameters; by contrast, there was a modest positive correlation between these parameters for lexical decision and speeded pronunciation response times. The conclusion that, for fixation durations, the degree of skew is uncorrelated with the location of the distribution's central tendency was also confirmed nonparametrically, by examining vincentile plots for subgroups of subjects. Finally, the stability of distributional parameters for a given subject was demonstrated to be relatively high. Taken together with previous findings of selective influence on the $\mu$ parameter of the fixation duration distribution, the present results suggest that in reading, the location and the skew of the fixation duration distribution may reflect functionally distinct processes. The authors speculate that the skew parameter may specifically reflect the frequency of processing disruption.
\end{abstract}

\section{Keywords Eye movements $\cdot$ Reading $\cdot$ Distribution fitting}

Both parametric and nonparametric methods have been used to investigate how experimental manipulations affect response time (RT) distributions (see Balota \& Yap, 2011, for a discussion). In particular, studies of visual word recognition have used ex-Gaussian fitting (Ratcliff, 1979) and vincentile plotting (Vincent, 1912) to investigate lexical decision (LDT), speeded pronunciation (or naming), and

\footnotetext{
A. Staub $(\bowtie) \cdot$ A. Benatar

Department of Psychology, University of Massachusetts, 430

Tobin Hall,

Amherst, MA 01003, USA

e-mail: astaub@psych.umass.edu
}

semantic categorization RTs (e.g., Andrews \& Heathcote, 2001; Balota \& Spieler, 1999; Balota, Yap, Cortese, \& Watson, 2008; Plourde \& Besner, 1997; Yap \& Balota, 2007). More recently, eye fixation durations in reading have been studied using the same methods (Staub, 2011; Staub, White, Drieghe, Hollway, \& Rayner, 2010; White \& Staub, 2012).

The present study is motivated by an apparent contrast between these single-word and eye movement studies. Although there are exceptions (e.g., Balota et al., 2008; Johnson, Staub, \& Fleri, 2012), it is generally the case in single-word tasks that experimental manipulations influence both the location of the distribution of RTs (the $\mu$ parameter of the ex-Gaussian distribution) and the weight of the distribution's right tail (the $\tau$ parameter). This is the case for manipulations of word frequency (e.g., Andrews \& Heathcote, 2001; Balota \& Spieler, 1999), stimulus quality (e.g., Plourde \& Besner, 1997; Yap \& Balota, 2007), and lexical repetition (Balota \& Spieler, 1999).

In the eye movement literature, the situation is somewhat different. Focusing on the duration of the first eye fixation on a critical word, Staub et al. (2010) found that word frequency affects both the $\mu$ and $\tau$ parameters. Reingold, Reichle, Glaholt, and Sheridan (2012) replicated this effect and also found that a manipulation of the validity of parafoveal preview (i.e., whether the word was fully visible in the parafovea, prior to direct fixation) influenced both parameters. However, four variables have been found to affect only $\mu$ : stimulus quality (whether text is presented normally or with diminished visual contrast; White \& Staub, 2012), predictability in context (i.e., cloze probability; Sheridan \& Reingold, 2012b; Staub, 2011), lexical ambiguity (Sheridan \& Reingold, 2012a), and the landing position of the eyes within the word (Reingold et al., 2012). In sum, of the six factors that have been investigated using distributional analysis of fixation durations, four appear to affect only $\mu$. 
White and Staub (2012) argued that selective influence on the $\mu$ parameter is not easily accommodated by current models of eye movements in reading (Engbert, Nuthmann, Richter, \& Kliegl, 2005; Reichle, Rayner, \& Pollatsek, 2003; cf. Carpenter \& McDonald, 2007). This selective influence suggests that the shift and skew of fixation duration distributions in reading may reflect functionally distinct underlying processes or mechanisms. If this conjecture is correct, it is also possible that these two parameters vary independently across subjects. Subjects who tend to have long fixations in general (i.e., a high $\mu$ parameter) may not show an especially pronounced right tail (a high $\tau$ parameter) and vice versa. The present study tests whether this is the case. We compare correlations between parameters in a large set of LDT and naming data (Yap, Balota, Sibley, \& Ratcliff, 2012) and in eye movement data from 153 subjects in five experiments. The central conclusion is that the $\tau$ parameter shows a modest positive correlation with $\mu$ and $\sigma$ in single-word tasks but these parameters are uncorrelated in eye movements. We then address the concern that this lack of correlation may be due to trade-offs in the fitting process, and we show that this is not the case. In addition, we establish that parameters of an individual subject's fixation duration distribution are relatively stable. We conclude by discussing a possible interpretation of the independence of the location and skew of the fixation duration distribution.

\section{Data sets}

LDT and naming data used for comparison with eye movement data were originally collected as part of the English Lexicon Project (http://elexicon.wustl.edu). Ex-Gaussian parameters for each subject ( $N=470$ for naming, $N=819$ for LDT) were computed by Yap et al. (2012). ${ }^{1}$

The eye movement data used in this study are firstfixation durations on target words in five experiments. Four have been previously reported: Drieghe, Rayner, and Pollatsek (2008) and White (2008), both of which were analyzed distributionally by Staub et al. (2010); Staub (2011); and White and Staub (2012). In the present study, we collapse across the within-subjects conditions in these experiments, obtaining a single distribution of fixation durations for each subject. (In one of the three conditions in White \& Staub, 2012, a single low-contrast word was presented within normal text. This condition elicited extremely long fixation durations, with unusual distributional characteristics, and is excluded from the present analysis. The condition in which the entire sentence was presented with low contrast is included here.) A total of 119 subjects

\footnotetext{
${ }^{1}$ Thanks to Melvin Yap for providing the ex-Gaussian fits. Yap et al. (2012) do not report the correlational analyses reported here.
}

were run in these four studies. Six subjects' data were not satisfactorily fit by the QMPE program (see below), with higher exit codes than other subjects and questionable parameter values (e.g., values of $\sigma$ near 0 ), leaving 113 usable subjects in the present study. The number of critical trials varied across these four studies from 78 to 120 , but due to word skipping and other data loss, on average, 77 usable first fixations were available.

The fifth experiment is a new study with a design similar to that in Staub (2011), although with somewhat more power. Each of 40 subjects read 170 critical sentences. These consisted of 85 item pairs, in which the same target word was either highly predictable or unpredictable in its context, on the basis of cloze norming. The apparatus and procedure were identical to those in Staub (2011). Predictability reduced mean first-fixation duration on the target word by $16 \mathrm{~ms}$ $(p<.01)$. As in Staub, this effect was due entirely to an effect on the $\mu$ parameter $(p<.01)$; the effect on the $\tau$ parameter was less than $1 \mathrm{~ms}$. Subjects had an average of 134 valid first fixations.

\section{Parameter estimation and correlations}

For each of the 153 eye movement subjects, ex-Gaussian parameters were estimated using the QMPE software (Cousineau, Brown, \& Heathcote, 2004; Heathcote, Brown, \& Mewhort, 2002). Fits were obtained as in Staub (2011) and White and Staub (2012). Table 1 provides the number of subjects, mean first-fixation duration, and the mean of the ex-Gaussian parameters for each of the five experiments. It is notable that the mean is substantially higher in Drieghe et al. (2008) and White (2008) than in the other experiments. Although this pattern should be interpreted with caution (the experiments were carried out on two continents, with a range of materials), a relevant difference is that the first two experiments used a Dual Purkinje Image eyetracker (Fourward Technologies), while the latter three used an Eyelink camera-based tracker (SR Research). This change in apparatus is accompanied by a change in the visual display, with the first two experiments presenting light text on a dark background, at low resolution, and the last three presenting dark text on a light background, at much higher resolution. There has been an informal consensus among researchers that fixation durations are shorter with this change in the physical characteristics of the display. Also notable is that this difference in the mean appears to be entirely captured by the $\mu$ parameter. If the visual display is indeed the critical difference between experiments, the conclusion that this affects only $\mu$ is consistent with White and Staub's finding that a within-subjects manipulation of visual contrast influences only $\mu$. 
Table 1 Mean first-fixation duration and mean of the best-fitting exGaussian parameters in each of the five eye movement experiments

\begin{tabular}{llllll}
\hline Experiment & $n$ & mean & mu & sigma & tau \\
\hline Drieghe et al. (2008) & 25 & 270 & 207 & 42 & 65 \\
White (2008) & 27 & 266 & 209 & 44 & 58 \\
Staub (2011) & 31 & 215 & 159 & 27 & 58 \\
White and Staub (2012) & 30 & 230 & 172 & 33 & 58 \\
Staub (2011) replication & 40 & 229 & 166 & 36 & 63 \\
\hline
\end{tabular}

Figure 1 presents scatterplots showing the relationship between each pair of ex-Gaussian parameters for the Yap et al. (2012) LDT and naming data for all 153 eye movement subjects, and separately for the 52 eye movement subjects who were tested with the Dual Purkinje Image tracker (light text) and the 101 who were tested with the Eyelink tracker (dark text). Correlations are reported on the plots. There is a significant positive correlation between $\mu$ and $\sigma$ in all data sets. However, while the $\tau$ parameter is positively correlated with the other two parameters in the LDT and naming data, there is no hint of a correlation in the eye movement data.

Schmiedek, Oberauer, Wilhelm, Süß, and Wittman (2007, Appendix A) have noted a potential problem for the interpretation of correlations between ex-Gaussian parameters. Their simulations demonstrated that due to fitting trade-offs, correlations between fitted values of $\mu$ and $\sigma$ with $\tau$ will tend to be negative when there is, in fact, no true correlation. This artifact would not explain the difference between the eye movement and single-word correlations, but it suggests that the true correlations in the eye movement data may be positive, with trade-offs reducing these correlations to zero.

We address this issue in two ways. First, we compute the correlation between the median of each subject's fixation duration distribution and the skewness of the distribution, using the standard skewness statistic:

$\gamma_{1}=\mu_{3} / \mu_{2}^{3 / 2}$

where $\mu_{3}$ is the distribution's third central moment and $\mu_{2}$ is the second central moment, or the variance. Positive values indicate rightward skew, and negative values indicate leftward skew; 151 of 153 skewness statistics were positive, as was expected. Overall, the correlation is $r=-.09(r=-.05$ for light text; $r=.08$ for dark text; all $p \mathrm{~s}>.2$ ). The overall negative correlation indicates that subjects with higher medians showed slightly less rightward skew. Thus, the lack of any positive correlation in the eye movement data between the central tendency of the distribution and the degree of skew holds in the absence of dependencies between parameters.

\section{Vincentile plots}

We also examine the relationship between a distribution's shift and skew visually, by means of vincentile plots. We construct plots assessing (1) whether subjects for whom $\mu$ is high do have shifted distributions, as compared with subjects for whom $\mu$ is low, while showing no difference in distributional skewing, and (2) whether subjects for whom $\tau$ is high do show a difference in skew, as compared with subjects for whom $\tau$ is low, while showing no distributional shift.

The plots are constructed as follows. Each subject's observations are divided into ten bins, such that the shortest $10 \%$ of the observations are in the first bin, the next shortest $10 \%$ in the next bin, and so on. The mean within each bin is then calculated. The mean of these subject means is then plotted, with the first vincentile (the shortest durations) on the left and the tenth vincentile on the right. Figure 2 presents these plots for all subjects (top row), for the subjects tested with light text (middle row), and for those tested with dark text (bottom row). Within each row, the left-hand plot compares the vincentiles for high- $\mu$ subjects (filled squares) and low- $\mu$ subjects (open squares), on the basis of a median split. Dividing subjects on the basis of $\mu$ results in two groups that differ substantially in distributional location but do not differ at all in the degree of skew. This is shown by the parallel curves in the plots. The center plot in each row compares the vincentiles for high- $\tau$ subjects (filled squares) and low- $\tau$ subjects (open squares). Dividing subjects on the basis of $\tau$ results in two groups that differ in the degree of skew but do not differ in distributional location. This is shown by the increasing difference between curves, moving across the vincentiles.

The right-hand plot in each row shows the difference at each vincentile between high- and low- $\mu$ subjects (open squares) and between high- and low- $\tau$ subjects (filled squares). The first of these curves is very flat, indicating that high- and low- $\mu$ subjects' vincentiles differ by about the same amount across the distribution, while the second of these curves is steeply sloped, indicating that high- and low- $\tau$ subjects differ primarily in the duration of long fixations.

The plot in the upper right can be compared with the simulated difference curves in Fig. 3. The open squares reflect differences based on 10,000 observations from two ex-Gaussian distributions with only a 47-ms difference in $\mu$ and a 12-ms difference in $\sigma$, corresponding to the observed differences in $\mu$ and $\sigma$ in the high- and low- $\mu$ groups. The filled squares reflect differences between two distributions with only 

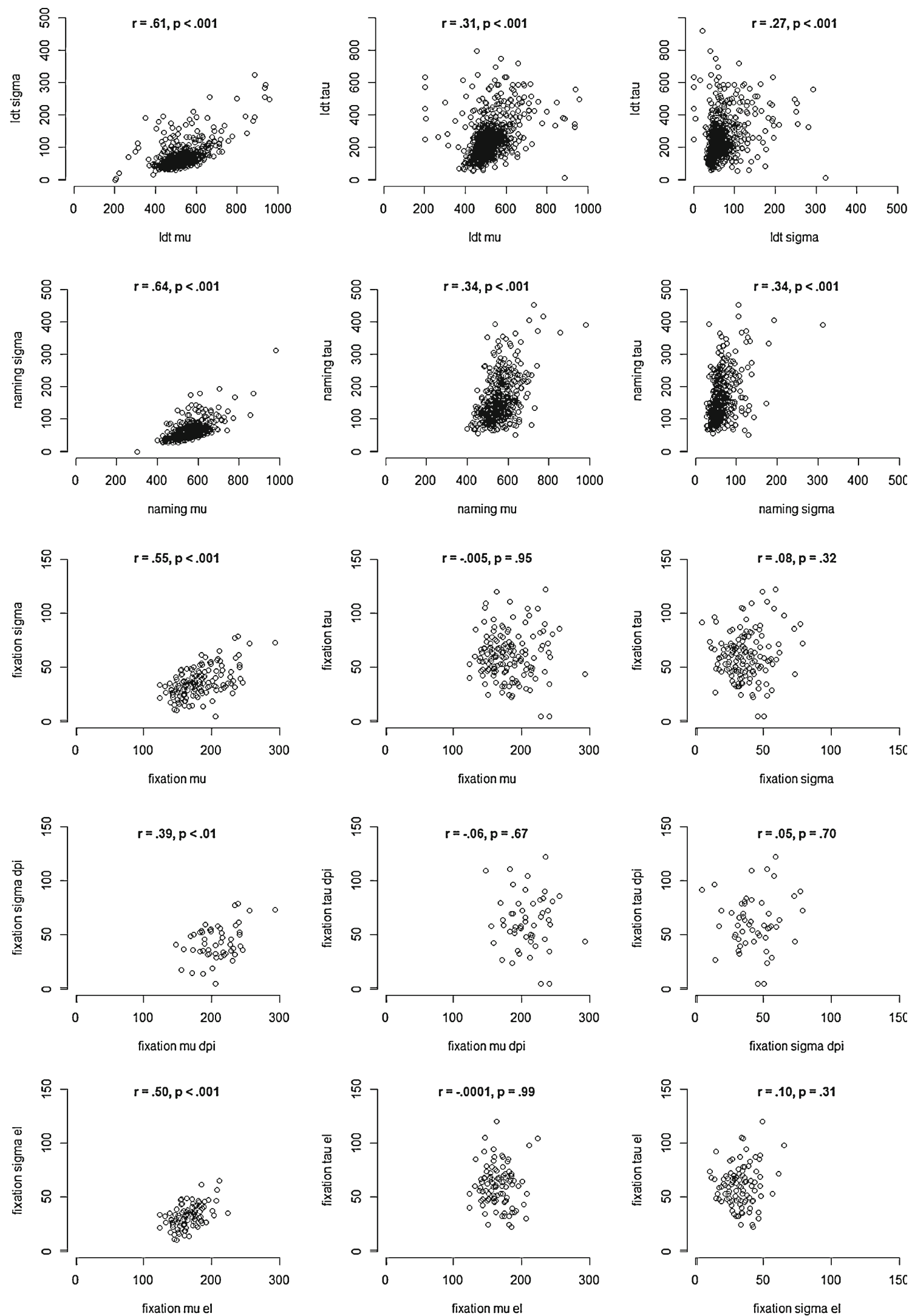

Fig. 1 Scatterplots for $\mu$ and $\sigma$ (left column), $\mu$ and $\tau$ (center column), and $\sigma$ and $\tau$ (right column) for each paradigm: LDT (top row); naming (second row); fixation durations, all experiments (third row); fixation durations, DPI experiments only (fourth row); and fixation durations, Eyelink experiments only (bottom row) 

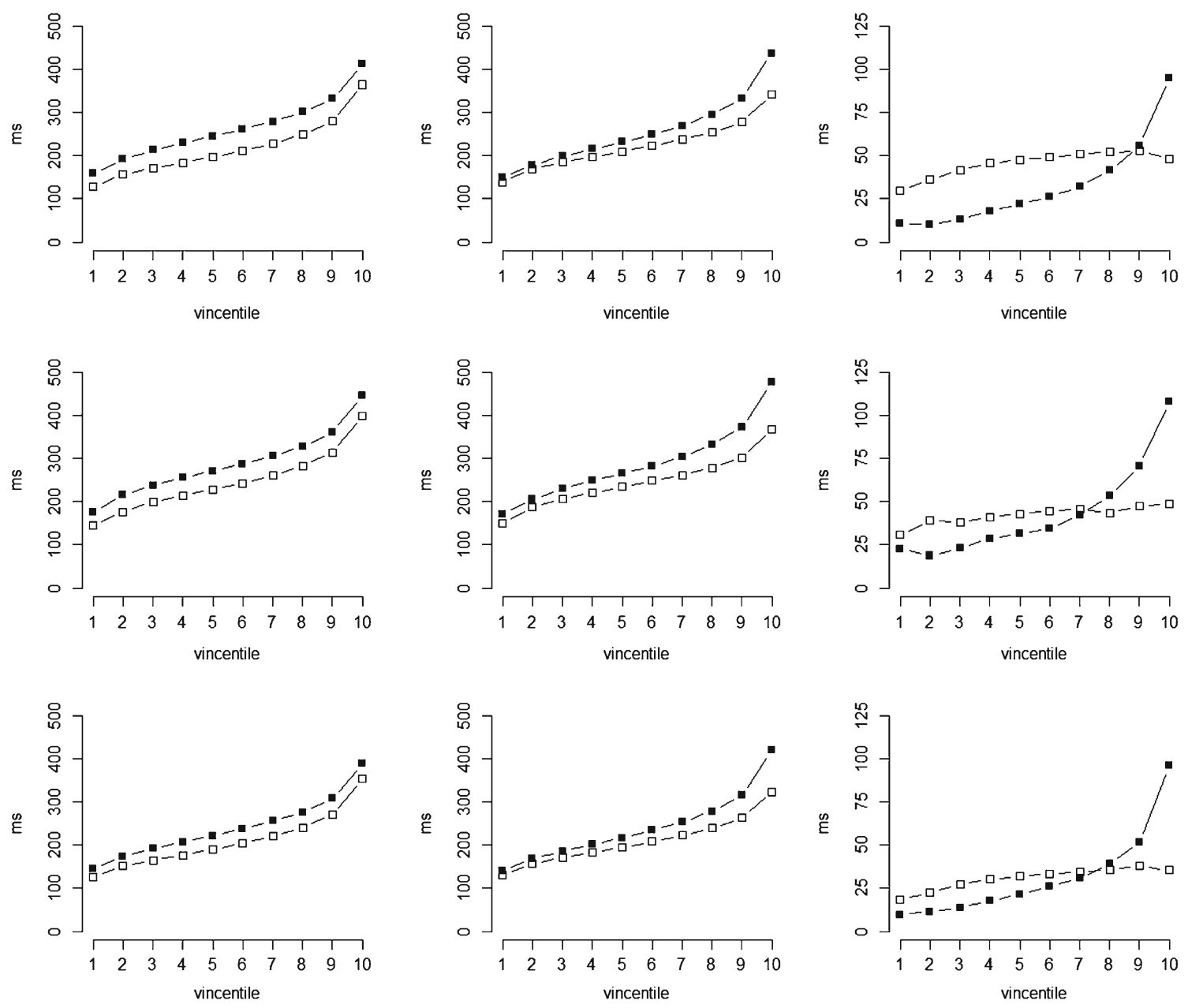

Fig. 2 Vincentile plots for high- and low- $\mu$ subjects (left column) and high- and low- $\tau$ subjects (center column), and difference plots based on these vincentiles (right column). Top row is for all subjects, middle row

a $32-\mathrm{ms}$ difference in $\tau$, corresponding to the observed difference in the high- and low- $\tau$ groups.

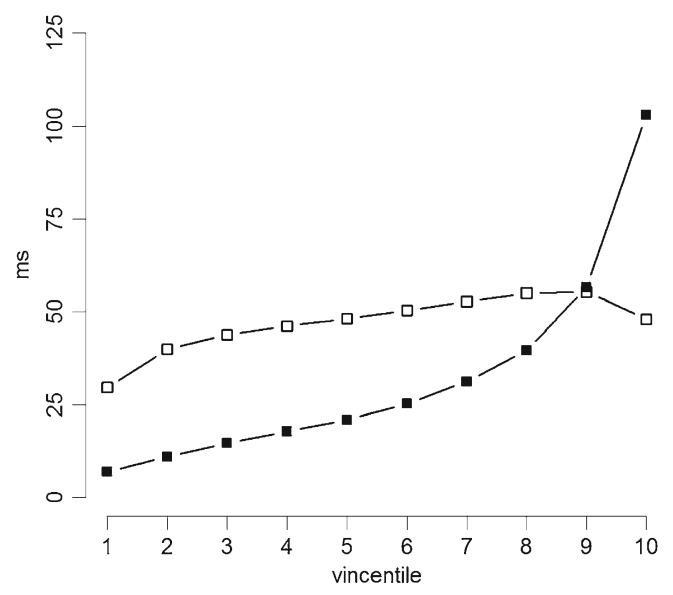

Fig. 3 Idealized vincentile plot for comparison to upper right plot in Fig. 2. Construction of this plot is described in the text is DPI experiments only, and bottom row is Eyelink experiments only. Futher details are provided in the text

\section{Stability of individual-subject parameters}

To assess whether individual eye movement subjects demonstrate stable distributional patterns, we further analyzed data from the 40 subjects in the new experiment reported above. For each subject, we obtained the best-fitting exGaussian parameters for the fixation immediately preceding fixation on the target word, which we refer to as the prior fixation, and for the first fixation on the material following the target word, which we refer to as the following fixation. Occasionally, there was no valid following fixation (e.g., when the target word was very near the end of the sentence), so fits were based on an average of 129 observations, rather than 134 .

The mean first-fixation duration on the critical word was strongly correlated with mean prior-fixation duration $(r=$ $.87, p<.001)$ and mean following-fixation duration $(r=.77$, $p<.001$ ). (Note that these correlations are across subjects; no conclusions can be drawn about correlations between successive fixations on individual trials.) Substantial 
positive correlations were also obtained between the exGaussian parameters for first-fixation duration and the corresponding parameters for these two distributions, as shown in Fig. 4. For example, the correlation between the firstfixation $\tau$ parameter and the prior-fixation $\tau$ parameter is $r=$ $.67(p<.001)$. Again, within each fixation duration distribution, there is a positive correlation between $\mu$ and $\sigma(r=$ $.48, p<.01$ for prior; $r=.48, p<.01$ for following), but not between $\mu$ and $\tau(r=-.08, p=.64$ for prior; $r=-.01, p=.96$ for following) or between $\sigma$ and $\tau(r=-.05, p=.78$ for prior; $r=-.03, p=.84$ for following).

To establish the upper limit on the correlations that could have been obtained in the preceding analysis, we fit the exGaussian to each of 40 bootstrap samples from each subject's first-fixation distribution. We then computed the correlation between the ex-Gaussian parameters fit to the original data and the parameters fit to each of the 40 bootstrap samples. The mean of these 40 correlations was .87 for $\mu, .80$ for $\sigma$, and .84 for $\tau$. Thus, while Yap et al. (2012) reported $r \mathrm{~s} \geq .92$ for parameters fit to odd and even trials in LDT and naming experiments (with approximately 3,374 LDT trials and 2,530 naming trials per subject), a correlation of this magnitude is clearly not possible in the present case. Indeed, the obtained correlations may be regarded as surprisingly high, given the theoretical maximum established by the bootstrap simulations.

\section{Discussion}

The degree to which a reader's fixation duration distribution is skewed appears to be uncorrelated with the location of the distribution. This conclusion has been established both parametrically and nonparametrically and stands in contrast to LDT and naming, for which there is a moderate, although highly significant, positive correlation between $\mu$ and $\tau$ values. The absence of correlation in the eye movement data is obtained despite substantial stability in an individual reader's ex-Gaussian fits.

The ex-Gaussian was at one time regarded as a cognitive model suggesting the contribution of two processes to RT, one with normally distributed finishing times and one with exponentially distributed finishing times (Hohle, 1965). This conception has fallen out of favor, however, and most researchers (including us) have regarded the ex-Gaussian simply as a statistical or descriptive model (for a discussion, see Heathcote, Popiel, \& Mewhort, 1991; Matzke \& Wagenmakers, 2009). But the dissociation between $\mu$ and $\tau$ in eye movement data, both in terms of experimental effects on the $\mu$ parameter only and in terms of the dissociation between parameters across subjects, suggests that there may indeed be two processes contributing to the location and skew of distributions of eye fixations in reading. In the remainder of this discussion, we suggest a very tentative account of what these two processes may be. (To be explicit, we are not suggesting that a fixation duration is the sum of finishing times of two distinct processes. Rather, we are suggesting that there may be distinct processes that influence the location of the fixation duration distribution and its skew, which are, in turn, reflected in the $\mu$ and $\tau$ parameters.)

We begin by considering which variables are known to influence the $\tau$ parameter. As was noted above, $\tau$ is increased when the fixated word is low in frequency (Staub et al., 2010) and when the word does not receive valid parafoveal preview (Reingold et al., 2012). However, $\tau$ is not increased when the fixated word is unpredictable in context (Staub, 2011) or lexically ambiguous (Sheridan \& Reingold, 2012a), and $\tau$ is not modulated by visual contrast (White \& Staub, 2012) or by the position of the eyes within the fixated word (Reingold et al., 2012).

The classification we propose in order to make sense of this pattern is based on a simple distinction between processing difficulty and processing disruption. (We note that this distinction is related to, but not identical to, the distinction between triggering and interference mechanisms discussed by Reingold et al., 2012.) We assume that normal perceptual and linguistic processing in reading may vary in its difficulty but that, in addition, there are episodes when normal processing does not succeed and some repair or recovery process follows. Such episodes are evident when the normal forward movement of the eyes is interrupted, but they may also occur within a single eye fixation. We suggest that a manipulation influences $\tau$ when it increases the likelihood of processing disruption, as opposed to simply modulating processing difficulty. On occasion, a reader may initially fail to recognize a low-frequency word or fail to access its meaning sufficiently to integrate it into the meaning of the sentence. Low-frequency words used in the experiments mentioned above are very low frequency indeed; for example, White (2008) used words such as adder, lout, and sari. Similarly, the change during a saccadic eye movement from one letter string (the invalid parafoveal preview) to another (the fixated word) may trigger processing disruption, as opposed to simply delaying the start of lexical processing of the fixated word. The other four manipulations, on the other hand, are unlikely to cause substantial disruption. For example, the unpredictable words used in these experiments are perfectly sensible in their sentence context, and the low-contrast sentences presented by White and Staub (2012) are still quite easy to read. In sum, we are proposing that the right tail of the fixation duration distribution consists of fixations that are lengthened due to processing disruption and that the degree to which the distribution is skewed reflects the relative frequency and/or severity of these disruptions. 

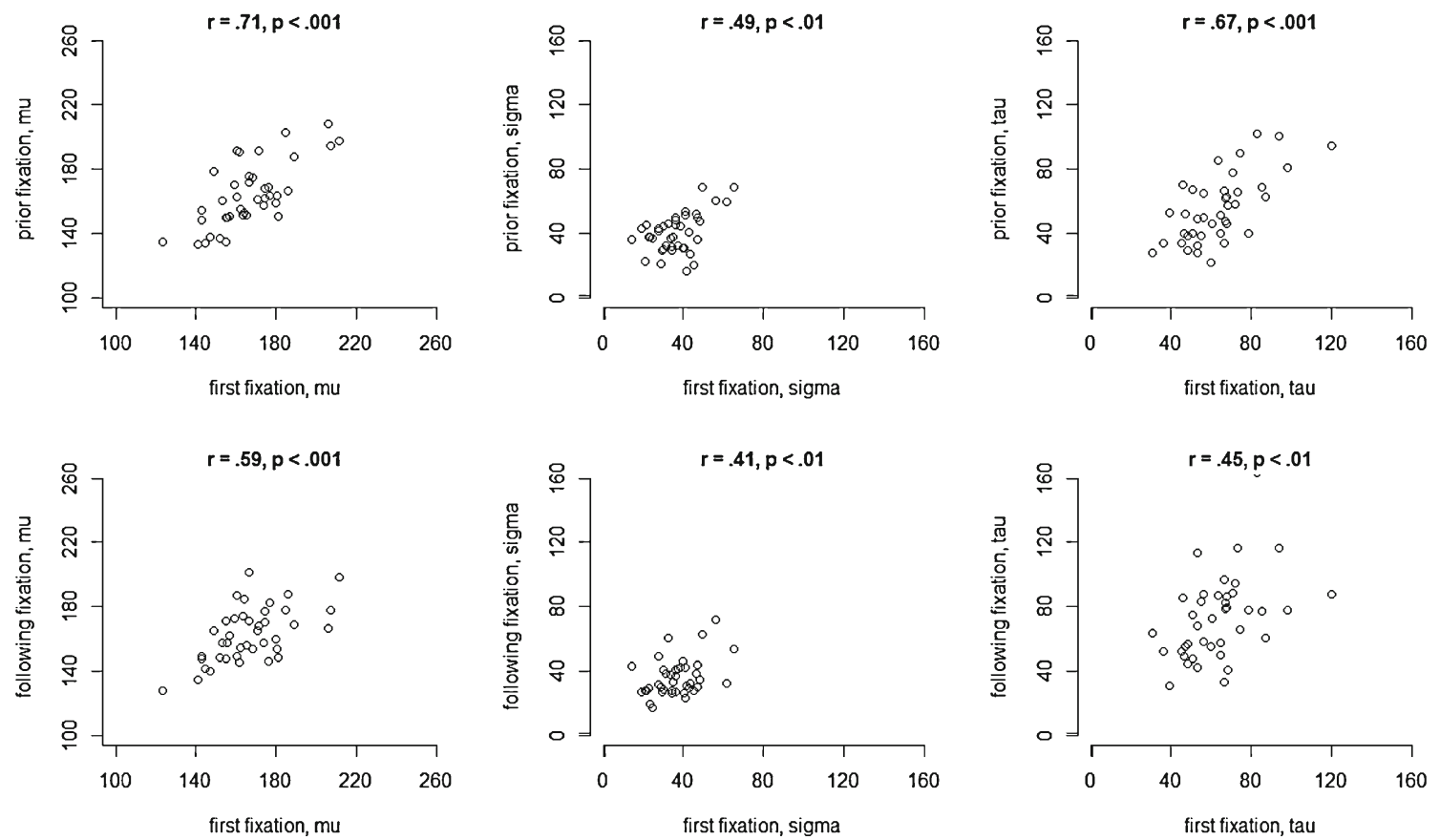

Fig. 4 Scatterplots showing relationships between ex-Gaussian parameters for first fixation and prior fixation (top row) and for first fixation and following fixation (bottom row). Left hand column is $\mu$, middle column is $\sigma$, and right-hand column is $\tau$

This hypothesis suggests that individual readers may vary independently on two dimensions. First, they vary in the time required for normal perceptual and linguistic processing during an eye fixation, which will determine the location of the fixation duration distribution. Second, they vary in how frequently normal processing is disrupted. Note that this frequency of disruption may vary, in part, as a function of a subject's criterion for modulating the usual fixation plan. Thus, individual differences in the $\mu$ parameter may reflect the difficulty of perceptual and linguistic processing, while individual differences in the $\tau$ parameter may reflect, in part or in their entirety, individual differences in a decision criterion. We do acknowledge previous individual-difference work (e.g., Kuperman \& Van Dyke, 2011) demonstrating effects of an individual reader's proficiency on both fixation durations and indices of disruption such as the frequency of regressive saccades. It remains to be determined in future research whether the independence observed here between the location and skew of a reader's fixation duration distribution is still observed across a wide range of reading proficiency.

The observed difference between tasks still remains to be explained; LDT and naming demonstrate a positive correlation between distributional location and skew. We note, however, that this task difference is, in fact, relatively slight. With correlations of just above 3 in LDT and naming, only about $10 \%$ of subject variation in $\tau$ is explained by variation in $\mu$. Given the extremely high reliability of the individualsubject parameter estimates obtained by Yap et al. (2012), this very modest relationship cannot be due to noisy parameter estimation. Thus, there is also a high degree of independence between $\mu$ and $\tau$ in LDT and naming data.

In sum, this study has shown that the location and skew of the distribution of a reader's eye fixation durations in reading are independent; a reader whose fixation durations are relatively long in general does not demonstrate an especially skewed distribution. Taken together with previous demonstrations of selective influence on one of these parameters, this suggests that the location and skew of the fixation duration distribution may reflect distinct aspects of visual and linguistic processing during reading.

\section{References}

Andrews, S., \& Heathcote, A. (2001). Distinguishing common and task-specific processes in word identification: A matter of some moment? Journal of Experimental Psychology: Learning, Memory, and Cognition, 27, 514-544.

Balota, D. A., \& Spieler, D. H. (1999). Word frequency, repetition, and lexicality effects in word recognition tasks: Beyond measures of central tendency. Journal of Experimental Psychology: General, 128, 32-55.

Balota, D. A., \& Yap, M. J. (2011). Moving beyond the mean in studies of mental chronometry: The power of response time distributional analyses. Current Directions in Psychological Science, 20, 160-166.

Balota, D. A., Yap, M. J., Cortese, M. J., \& Watson, J. M. (2008). Beyond mean response latency: Response time distributional analyses of semantic priming. Journal of Memory \& Language, $59,495-523$.

Carpenter, R. H. S., \& McDonald, S. A. (2007). LATER predicts saccade latency distributions in reading. Experimental Brain Research, 177, 176-183. 
Cousineau, D., Brown, S., \& Heathcote, A. (2004). Fitting distributions using maximum likelihood: Methods and packages. Behavior Research Methods, Instruments, \& Computers, 36, 742-756.

Drieghe, D., Rayner, K., \& Pollatsek, A. (2008). Mislocated fixations can account for parafoveal-on-foveal effects in eye movements during reading. Quarterly Journal of Experimental Psychology, $61,1239-1249$.

Engbert, R., Nuthmann, A., Richter, E. M., \& Kliegl, R. (2005). SWIFT: A dynamical model of saccade generation during reading. Psychological Review, 112, 777-813.

Heathcote, A., Brown, S., \& Mewhort, D. J. K. (2002). Quantile maximum likelihood estimation of response time distributions. Psychonomic Bulletin \& Review, 9, 394-401.

Heathcote, A., Popiel, S. J., \& Mewhort, D. J. K. (1991). Analysis of response time distributions: An example using the Stroop task. Psychological Bulletin, 109, 340-347.

Hohle, R. (1965). Inferred components of reaction times as functions of foreperiod duration. Journal of Experimental Psychology, 69, 382-386.

Johnson, R. L., Staub, A., \& Fleri, A. M. (2012). Distributional analysis of the transposed-letter neighborhood effect on naming latency. Journal of Experimental Psychology: Learning, Memory, and Cognition, 38, 1773-1779.

Kuperman, V., \& Van Dyke, J. A. (2011). Effects of individual differences in verbal skills on eye-movement patterns during sentence reading. Journal of Memory \& Language, 65, 45-73.

Matzke, D., \& Wagenmakers, E.-J. (2009). Psychological interpretation of the ex-Gaussian and shifted Wald parameters: A diffusion model analysis. Psychonomic Bulletin \& Review, 16, 798-817.

Plourde, C. E., \& Besner, D. (1997). On the locus of the word frequency effect in visual word recognition. Canadian Journal of Experimental Psychology, 51, 181-194.

Ratcliff, R. (1979). Group reaction time distributions and an analysis of distribution statistics. Psychological Bulletin, 86, 446-461.

Reichle, E. D., Rayner, K., \& Pollatsek, A. (2003). The E-Z Reader model of eye-movement control in reading: Comparisons to other models. Behavioral and Brain Sciences, 26, 445-526.

Reingold, E. M., Reichle, E. D., Glaholt, M. G., \& Sheridan, H. (2012). Direct lexical control of eye movements in reading: Evidence from a survival analysis of fixation durations. Cognitive Psychology, 65, 177-206.

Schmiedek, F., Oberauer, K., Wilhelm, O., Süß, H.-M., \& Wittman, W. W. (2007). Individual differences in components of reaction time distributions and their relations to working memory and intelligence. Journal of Experimental Psychology: General, 136, 414 429.

Sheridan, H., \& Reingold, E. M. (2012a). The time course of contextual influences during lexical ambiguity resolution: Evidence from distributional analysis of fixation durations. Memory \& Cognition, 40, 1122-1131.

Sheridan, H., \& Reingold, E. M. (2012b). The time course of predictability effects in reading: Evidence from a survival analysis of fixation durations. Visual Cognition, 20, 733-745.

Staub, A. (2011). The effect of lexical predictability on distributions of eye fixation durations. Psychonomic Bulletin \& Review, 18, 371376.

Staub, A., White, S. J., Drieghe, D., Hollway, E. C., \& Rayner, K. (2010). Distributional effects of word frequency on eye fixation durations. Journal of Experimental Psychology: Human Perception and Performance, 36, 1280-1293.

Vincent, S. B. (1912). The function of the viborissae in the behavior of the white rat. Behavioral Monographs, 1, No. 5.

White, S. J. (2008). Eye movement control during reading: Effects of word frequency and orthographic familiarity. Journal of Experimental Psychology: Human Perception and Performance, 34, 205-223.

White, S. J., \& Staub, A. (2012). The distribution of fixation durations during reading: Effects of stimulus quality. Journal of Experimental Psychology: Human Perception \& Performance, 38, 603-617.

Yap, M. J., \& Balota, D. A. (2007). Additive and interactive effects on response time distributions in visual word recognition. Journal of Experimental Psychology: Learning, Memory, \& Cognition, 33, 274-295.

Yap, M. J., Balota, D. A., Sibley, D. E., \& Ratcliff, R. (2012). Individual differences in visual word recognition: Insights from the English Lexicon Project. Journal of Experimental Psychology: Human Perception and Performance, 38, 53-79. 